\title{
A parameterization of the diffuse transmittance and reflectance for aerosol remote sensing problems
}

\author{
A.A. Kokhanovsky ${ }^{\mathrm{a}, \mathrm{b}, *}$, B. Mayer ${ }^{\mathrm{c}}$, V.V. Rozanov ${ }^{\mathrm{a}}$ \\ a Institute of Environmental Physics, Bremen University, Otto Hahn Allee 1, D-28334 Bremen, Germany \\ ${ }^{\mathrm{b}}$ Institute of Physics, 70 Skarina Avenue, Minsk 220072, Belarus \\ ${ }^{\mathrm{c} I n s t i t u t e}$ of Atmospheric Physics, DLR Oberpfaffenhofen, D-82234 Wessling, Germany
}

Received 5 January 2004; accepted 25 July 2004

\begin{abstract}
To account for surface reflection is a major and most complex issue of any satellite aerosol retrieval algorithm. The surface contribution to the satellite signal is usually calculated assuming Lambertian reflection. Then the problem is reduced to the calculation of total atmospheric transmittance and spherical albedo. Usually, these parameters are stored in so-called look-up tables (LUTs), which can be quite large. The main aim of this paper is the parameterization of these look-up tables in terms of simple approximate equations, which can be subsequently used in aerosol remote sensing techniques over reflecting land surfaces.
\end{abstract}

(C) 2004 Elsevier B.V. All rights reserved.

Keywords: Radiative transfer; Aerosols; Clouds

\section{Introduction}

The accuracy of aerosol remote sensing techniques heavily depends on the information on the ground reflectance. It is usually assumed that the ground can be approximated by a Lambertian surface, which simplifies the calculations to large extent. In particular, the

* Corresponding author. Tel.: + 49421218 4475; fax: +49 4212184555 .

E-mail address: alexk@iup.physik.uni-bremen.de (A.A. Kokhanovsky). 
account for the Lambertian ground reflection in aerosol remote sensing problems can be done using standard equations (Sobolev, 1972):

$$
R=R_{b}+\frac{A t_{1} t_{2}}{1-r A}, \quad T=T_{b}+\frac{A t_{1} \mathcal{R}}{1-r A}
$$

valid for homogeneous light scattering media. Here $R$ is the aerosol reflection function, $T$ is the aerosol transmission function, $R_{b} \equiv R(A=0), T_{b} \equiv T(A=0), A$ is the ground albedo, $r$ is the aerosol spherical albedo, $t_{1}$ is the total (diffuse + direct) atmospheric transmittance from the sun to the surface and $t_{2}$ is the total transmittance from the surface to the satellite. $\mathcal{R}$ is the diffuse reflectance in the direction of the satellite which approaches $\mathcal{R}=1-t_{2}$ for nonabsorbing aerosol. The exact definition of all parameters and functions in Eq. (1) is given by Sobolev (1972).

Usually $R_{b}$ and $T_{b}$ are tabulated (after subtraction of the Rayleigh contribution). Then the aerosol optical thickness $\tau$ can be retrieved from the look-up tables for a given observation geometry, and prescribed aerosol phase function and single scattering albedo $\omega_{0}$. It is often assumed that the single scattering albedo is close to 1 . For a non-zero surface albedo, one has to account for the contribution of the surface reflection, which is given by the second terms in Eq. (1). The value of $A$ is not known; hence, an a-priori assumption of the spectral surface albedo $A(\lambda)$ is required. There are also possibilities to tune this assumed spectrum as proposed by Von Hoyningen-Huene et al. (2003). In particular, $t_{1}, t_{2}, r$, and $\mathcal{R}$ are tabulated for various observation/sun geometries, aerosol single scattering albedos, optical thicknesses and phase functions. This leads to rather large, multi-dimensional databases. The aim of this paper is the reduction of these look-up tables (LUTs) by developing a parameterization based on exact radiative transfer calculations. Such a parameterization allows a fast online calculation of $t_{1}, t_{2}, r$, and $\mathcal{R}$ for exactly the required conditionsavoiding the need to pre-calculate extensive databases and to interpolate between the stored grid points. Only the case of nonabsorbing media is considered. The uncertainty of the proposed parameterization is well below the uncertainty of the spectral surface albedo $A(\lambda)$. Hence the error is negligible as far as practical aspects of passive aerosol remote sensing from space are of concern. The equations can also be used for back-of-envelope calculations of the surface influence on atmospheric reflectance and transmittance.

\section{The parameterization of total transmittance and spherical albedo}

In a first step, diffuse transmittance and spherical albedo were calculated with the libRadtran radiative transfer package developed by Kylling and Mayer (1993-2004). LibRadtran includes a detailed atmospheric model, with molecules, aerosols, as well as water and ice clouds, which can be defined and modified in a user-friendly and flexible way. The package provides different numerical solvers of the radiative transfer equation, including exact discrete-ordinate schemes, a fast two-stream code, a polarizationdependent solver, and even a fully three-dimensional Monte Carlo code for horizontally inhomogeneous problems. LibRadtran has been extensively validated by comparison with other radiative transfer schemes (e.g. Van Weele et al., 2000) and by comparison with observations (e.g. Mayer et al., 1997). For the particular application presented here, the 
discrete ordinate solver by Stamnes et al. (1988), version 2.0, was chosen which basically allows exact calculation of radiances and irradiances. The results obtained were compared with those derived from the SCIATRAN (Rozanov et al., 2002) radiative transfer code. The differences were found to be smaller than $1 \%$, which confirms the high accuracy of both radiative transfer solvers.

The commonly used Henyey-Greenstein scattering phase function was chosen for the aerosol which is an analytical function of the asymmetry parameter $g$ :

$$
p(\theta)=\frac{1-g^{2}}{\left(1+g^{2}-2 g \cos \theta\right)^{1.5}} .
$$

Here $\theta$ is the scattering angle. Eq. (2) is only a rough approximation of a real aerosol scattering phase function but this is only of minor importance for our application because diffuse transmittance and reflectance are mostly governed by the value of the asymmetry parameter $g$ and not by the precise angular structure of the phase function.

We propose the following parameterizations based on the exact radiative transfer calculations performed for $\omega_{0}=1$ :

$$
\begin{aligned}
& r=\tau(a \exp (-\tau / \alpha)+b \exp (-\tau / \beta)+c), \\
& t_{j}=\exp \left(-\tau / \mu_{j}\right)+\tau \exp \left(-u_{j}-v_{j} \tau-w_{j} \tau^{2}\right),
\end{aligned}
$$

The second term in Eq. (4) gives the diffuse transmittance. Here $\mu_{1}$ is the cosine of the solar angle, $\mu_{2}$ is the cosine of the satellite angle, and

$$
\begin{aligned}
& u_{j}=\sum_{m=0}^{3} h_{m} \mu_{j}^{m}, \\
& v_{j}=p_{0}+p_{1} \exp \left(-p_{2} \mu_{j}\right), \\
& w_{j}=q_{0}+q_{1} \exp \left(-q_{2} \mu_{j}\right) .
\end{aligned}
$$

The constants $a, b, c, \alpha, \beta, p_{0}, q_{0}, p_{1}, q_{1}, p_{2}, q_{2}, h_{0}, h_{1}, h_{2}, h_{3}$ are parameterized using polynomial expansions with respect to the asymmetry parameter $g$ of the HenyeyGreenstein phase function. The standard regression routine incorporated in the ORIGIN package has been used for the parameterization. For all constants a third order polynomial is used (except for $h_{0}$ where a fourth order polynomial is required), e.g.

$$
a=\sum_{s=0}^{3} a_{s} g^{s}
$$

for the coefficient $a$. Coefficients for all parameters are given in Table 1. Note that Eqs. (3) and (4) provide the correct behavior for $r, t_{j}$ in the limit $\tau \rightarrow 0$ : In this case $r \rightarrow 0$ and $t_{j} \rightarrow i$.

The parameterization is valid for $g \in[0,0.9], \mu_{j} \in[0.2,1.0]$, and $\tau \in[0,2]$. This covers the usual variability of aerosol optical thickness and observation geometries. The asymmetry parameter of aerosol media is typically close to 0.7 (Kokhanovsky, 2004). Our technique 
Table 1

List of constants for Eqs. (3)-(8) $\left(h_{04}=-2.3\right)$

\begin{tabular}{lcccc}
\hline$s$ & 0 & 1 & 2 & 3 \\
\hline$a_{s}$ & 0.18016 & -0.18229 & 0.15535 & -0.14223 \\
$b_{s}$ & 0.58331 & -0.50662 & -0.09012 & 0.0207 \\
$\alpha_{s}$ & 0.16775 & -0.06969 & 0.08093 & -0.08903 \\
$\beta_{s}$ & 1.09188 & 0.08994 & 0.49647 & -0.75218 \\
$c_{s}$ & 0.21475 & -0.1 & 0.13639 & -0.21948 \\
$h_{0 s}$ & -1.88227 & 0.53661 & -1.8047 & 3.26348 \\
$h_{1 s}$ & 5.97763 & -2.04621 & -2.0173 & 1.44843 \\
$h_{2 s}$ & -5.47825 & 2.42154 & -3.37057 & 6.13805 \\
$h_{3 s}$ & 2.07593 & -2.03761 & 6.25975 & -7.35503 \\
$p_{0 s}$ & 0.4923 & 1.0471 & -2.61112 & 1.53155 \\
$p_{1 s}$ & 4.01521 & -0.25886 & -2.85378 & 3.61515 \\
$p_{2 s}$ & 3.76447 & 3.29106 & -12.37951 & 9.85 \\
$q_{0 s}$ & 0.000076 & -0.316 & 0.67744 & -0.4093 \\
$q_{1 s}$ & -1.31136 & -0.8901 & 3.55 & -3.0646 \\
$q_{2 s}$ & 5.21931 & 7.2255 & -23.43878 & 17.65629 \\
\hline
\end{tabular}

can be applied not only to aerosol media but also to thin clouds ( $g \approx 0.75-0.85$ ) and to the case of isotropic scattering $(g=0)$. Parameterizations for optically thick media are given in a separate publication (Kokhanovsky and Mayer, 2003). For most cases, errors of the parameterization are not larger than $2 \%$, which is satisfactory for most aerosol remote sensing problems (especially taking into account other uncertainties involved). A detailed study of the accuracy of Eqs. (3) and (4) is given in the next section.

\section{The accuracy of the parameterization}

Let us consider the accuracy of the proposed parameterization for the spherical albedo $r$ (see Fig. 1a). The spherical albedo strongly depends on the asymmetry parameter g. E.g., it can reach 0.15 for molecular scattering at $\tau=0.2$. It is around 0.35 for heavy aerosol loads with $\tau=2.0$ and $g=0.7$. The error of Eq. (3) for the spherical albedo $r$ is rather small, see Fig. 1b. In particular, it is smaller than $2 \%$ for $\tau \in[0.01,2.0]$ and $g \in[0.0,0.9]$. A parameterization of the spherical albedo $r$ for larger values of $\tau$ is given by Kokhanovsky and Mayer (2003).

The total transmittance $\mathrm{t}$ is given in Fig. $2 \mathrm{a}$ as function of $\tau$ at $g=0.7$ and several values of $\mu$. The error in the total transmittance $t$ is presented in Fig. 2b. We see that the errors are smaller than $2 \%$ for most cases. In particular, for $g=0.7$, it is smaller than $3 \%$ for $\mu>0.3$. Note that the vertical spread in Fig. $2 b$ is due to different angles and that in Fig. $2 \mathrm{c}$ is due to various optical thicknesses from 0 to 2.0. We conclude that the maximum error is around $8 \%$ for $g \in[0.0,0.9], \tau \in[0,2], \mu \in[0.2,1.0]$. The error is smaller than $4 \%$ for $\tau \leq 1.6$, $g \leq 0.8, \mu \in[0.2,1.0]$. This is the most important range of parameters as far as aerosol remote sensing is concerned.

Because of the relation $\mathcal{R}=1-t_{2}$ valid for nonabsorbing media, the errors of our parameterization for $\mathcal{R}$ can be found from those given in Fig. $2 \mathrm{~b}$ for the value of $t_{2}$. In particular, we have: $\delta_{\mathcal{R}}=-K \delta_{t_{2}}$, where $K=t_{2} / \mathcal{R}, \delta_{\mathcal{R}}$ is the relative error in the value of 

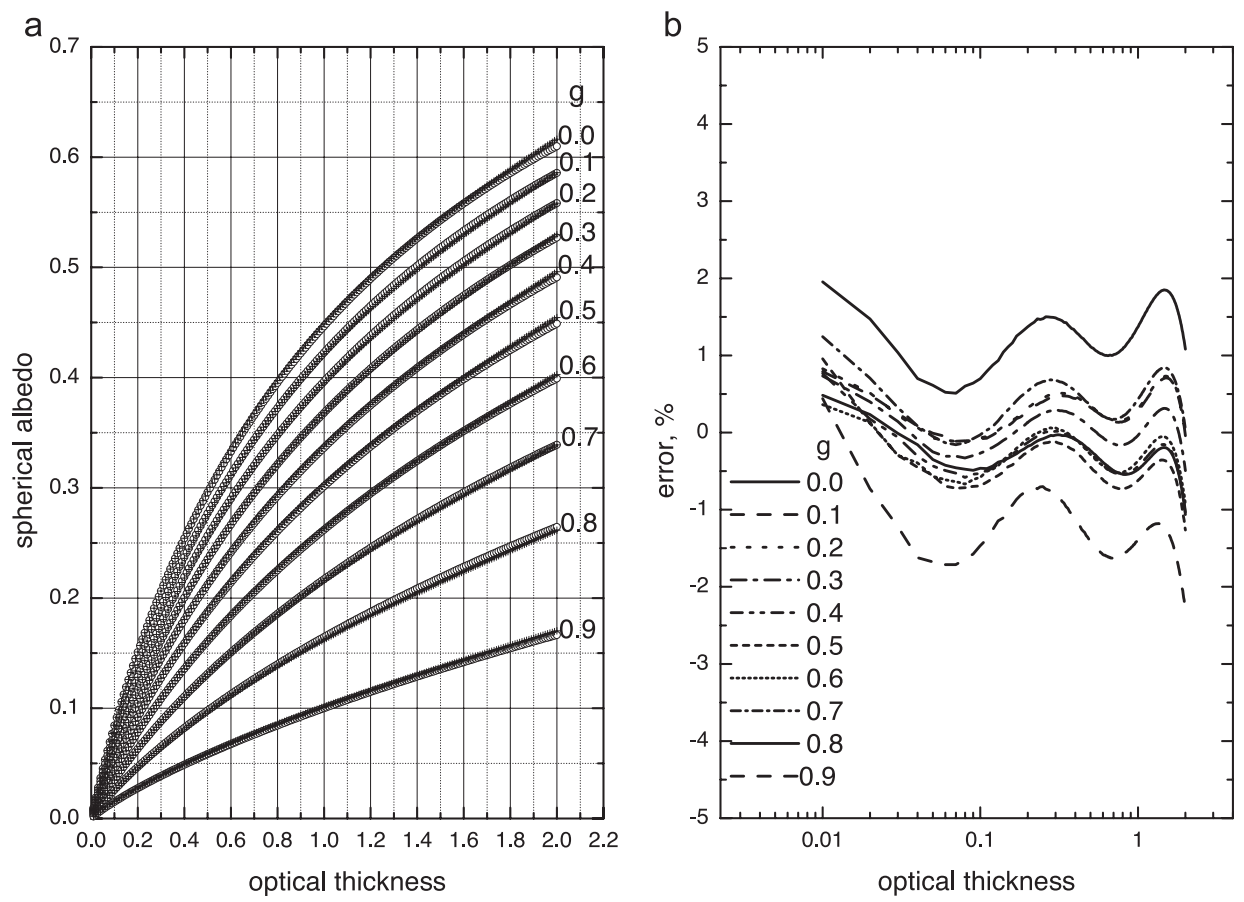

Fig. 1. (a) The spherical albedo as function of optical thickness for the Henyey-Greenstein phase function with various values of $g=0.0-0.9$. Crosses give the results of our parameterization, circles are obtained by exact radiative transfer calculations with $\omega_{0}=1$. (b) The relative error of Eq. (3) in \% for various values of $g$.

$\mathcal{R}$, and $\delta_{t_{2}}$ is the relative error in the value of $t_{2}$. We found that $\delta_{\mathcal{R}}$ is generally smaller than $5 \%$ (except, at $\mu=1$, where $\mathcal{R}$ is rather small and, therefore, $K \approx 3.0\left|\delta_{\mathcal{R}}\right| \approx 10 \%$ ).

The relative error $\delta_{R}$ in the reflection function $R$ (see Eq. (1)) is given as follows:

$$
\delta_{R}=M \sqrt{\delta_{t_{1}}^{2}+\delta_{t_{2}}^{2}+N^{2} \delta_{r}^{2}},
$$

where $M=R_{\mathrm{S}} / R, R_{s}=A t_{1} t_{2} /(1-r A), N=r A /(1-r A)$. The error decreases with $A$ as one might expect. The relative error in the transmission function $T$ can be determined with a similar expression. Then one needs to substitute $t_{2}$ by $\mathcal{R}$ in Eq. (9).

\section{Conclusion}

Simple analytical equations for the spherical albedo, diffuse and total transmittance of aerosol media are given. A parameterization has been developed based on exact radiative transfer calculations for a homogeneous nonabsorbing layer characterized by the HenyeyGreenstein phase function.

The parameterization can be used in aerosol remote sensing algorithms (Von Hoyningen-Huene et al., 2003). It can be applied also to related atmospheric research problems (e.g. for ocean-color algorithms; see e.g. Santer and Martiny, 2003). The 


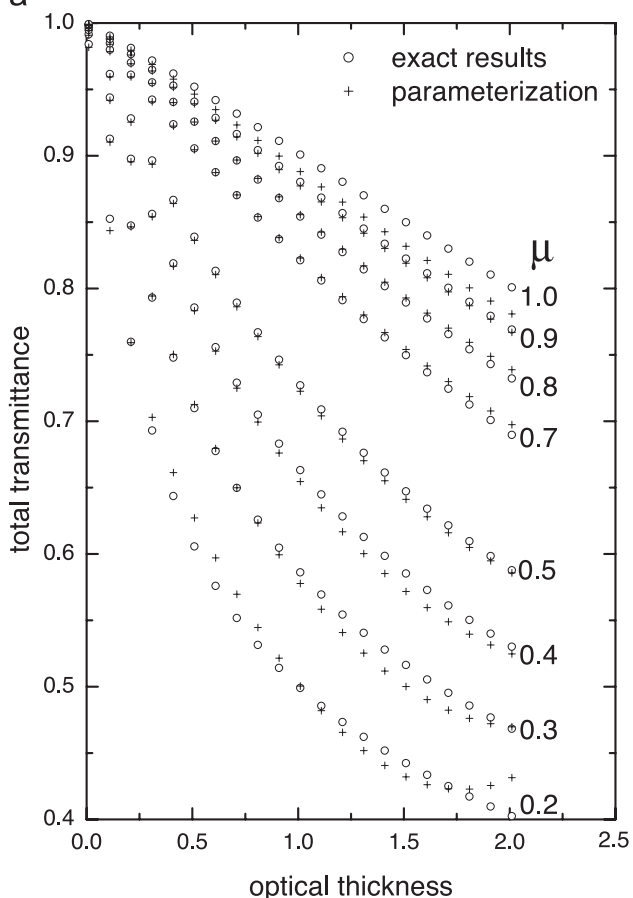

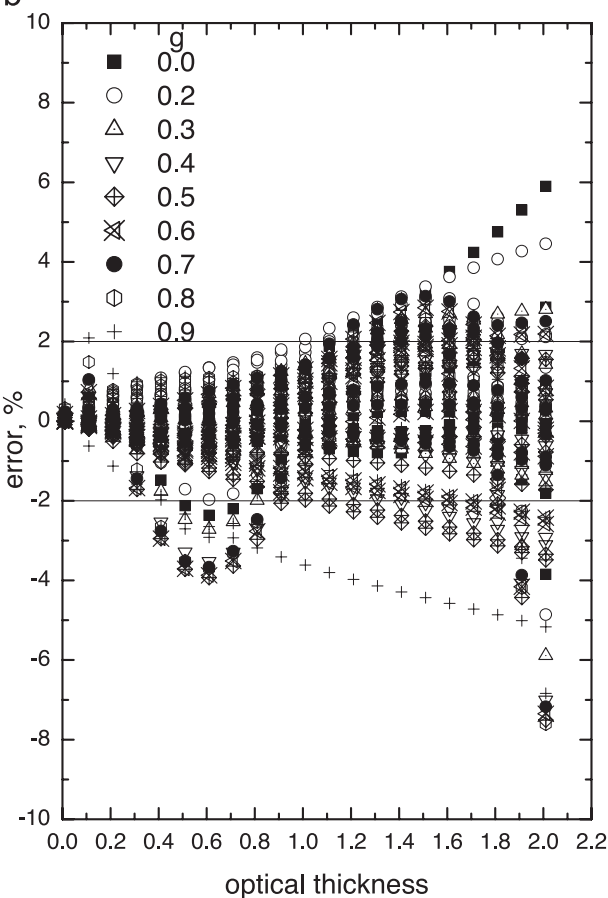

C

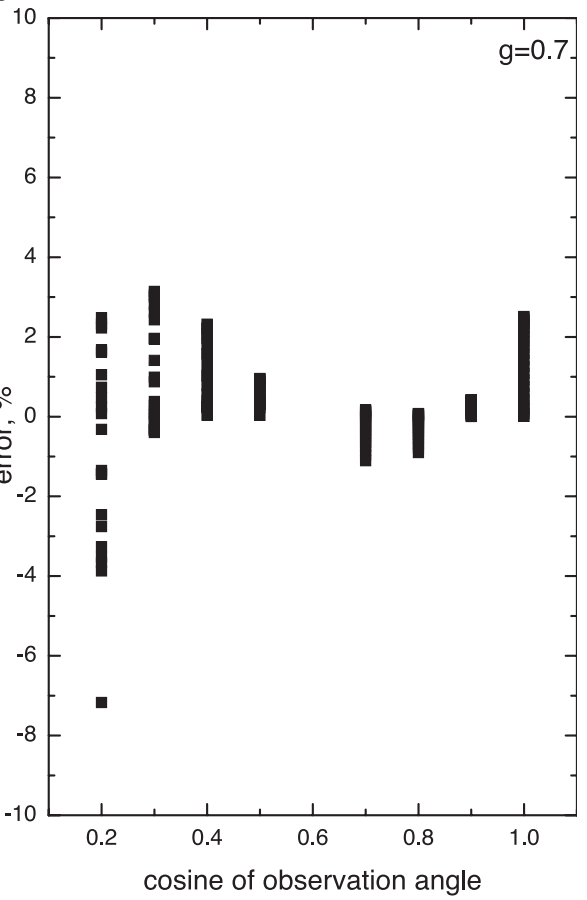

Fig. 2. (a) The total transmittance as function of the optical thickness for the Henyey-Greenstein scattering phase function with $g=0.7, \omega_{0}=1$, and various values of $\mu=0.2-$ 1.0. Crosses give the results of the parameterization, circles are obtained from exact radiative transfer calculations for nonabsorbing media. (b) The relative error of Eq. (4) in $\%$ for various values of $g=0.0-0.9$ and $\mu=0.2-1.0$. The vertical spread of data is due to various angles $\mu$. (c) The relative error of Eq. (4) in $\%$ at $g=0.7$ as function of the cosine of the observation angle $\mu$. The vertical spread is due to the variation of the error with optical thickness. The largest errors occur for large solar and satellite observation angles. Such cases are rarely used in aerosol remote sensing problems. 
uncertainty of the parameterization is typically below $2 \%$, which is accurate enough for most of applications.

\section{Acknowledgements}

The work was supported by the DFG Projects BU 688/8-1 and MA2458/1-1.

\section{References}

Kokhanovsky, A.A., 2004. Light Scattering Media Optics: Problems and Solutions. Springer-Praxis, Berlin.

Kokhanovsky, A.A., Mayer, B., 2003. Light reflection and transmission by nonabsorbing turbid slabs: simple approximations. J. Opt. A, Pure Appl. Opt. 5, 43-46.

Kylling, A., Mayer, B., 1993-2004. The libRadtran package. Freely available and documented at http:// www.libradtran.org; publication in preparation.

Mayer, B., Seckmeyer, G., Kylling, A., 1997. Systematic long-term comparison of spectral UV measurements and UVSPEC modeling results. J. Geophys. Res. 102 (D7), 8755-8767.

Rozanov, V.V., Buchwitz, M., Eichmann, K.-U., de Beek, R., Burrows, J.P., 2002. SCIATRAN—a new radiative transfer model for geophysical applications in the 240-2400 $\mathrm{nm}$ spectral range: the pseudo-spherical version. Adv. Space Res. 29, $1831-1835$.

Santer, R., Martiny, N., 2003. Sky-radiance measurements for ocean-color calibration-validation. Appl. Opt. 42, $896-907$

Sobolev, V.V., 1972. Light Scattering in Planetary Atmospheres. Nauka, Moscow.

Stamnes, K., Tsay, S.C., Wiscombe, W., Jayaweera, K., 1988. A numerically stable algorithm for discreteordinate-method radiative transfer in multiple scattering and emitting layered media. Appl. Opt. 27 (12), 2502-2509.

Van Weele, M., Martin, T.J., Blumthaler, M., Brogniez, C., den Outer, P.N., Engelsen, O., Lenoble, J., Pfister, G., Ruggaber, A., Walravens, B., Weihs, P., Dieter, H., Gardiner, B.G., Gillotay, D., Kylling, A., Mayer, B., Seckmeyer, G., Wauben, W., 2000. From model intercomparisons towards benchmark UV spectra for six real atmospheric cases. J. Geophys. Res. 105 (D4), 4915-4925.

Von Hoyningen-Huene, W., Freitag, M., Burrows, J.P., 2003. Retrieval of aerosol optical thickness over land surfaces from top-of-atmosphere radiance. J. Geophys. Res. 108 (D1), 4008, doi:10.1029/2001JD001543. 